\title{
Editorial
}

\section{Narcotics and myocar- dial performance in patients with coronary artery disease}

The article by Miller et al. ' in this issue of the Canadian Journal of Anaesthesia reports the results of a complex clinical study using sophisticated techniques in a large number of patients. Few investigators have the determination or resources to complete such a study. Both the methods and the findings of this work merit careful consideration by all anaesthetists caring for patients with heart discase. I will summarize the report of Miller $e t$ al. and then discuss the use of pressure-volume loops to analyze left ventricular function. The reader will see this powerful technique used in anaesthesia research with increasing frequency. Next, the need to define carefully what is meant by myocardial depression will be emphasized. Finally, a question will be raised that must always be addressed when assessing the results of a clinical study: can I apply these findings to my own practice?

The study was designed to examine the cardiovascular effects of inducing anaesthesia in patients with coronary artery disease using three different narcotics. Ninety-six patients scheduled for elective coronary artery surgery were assigned randomly to keceive fentanyl, sufentanil, or alfentanil for anaesthetic induction. Using a nuclear probe to estimate ejection fraction and a pulmonary artery catheter to determine cardiac output and from this stroke volume, the authors were able to calculate left ventricular end-diastolic and end-systolic volumes. Myocardial performance was described by the change in stroke work index associated with an increase in end-diastolic volume. Left ventricular systolic function (the ability of the heart to empty) was described by the end-systolic pressure-volume relation (discussed below).

Diastolic left ventricular compliance was determined by plotting the change in pulmonary capillary wedge pressure with increases in end-diastolic volume. In nine

Department of Anaesthesia, H2, Room 211, University of Ottawa Heart Institute, Ottawa Civic Hospital, 1053 Carling Avenue, Ottawa, Ontario, K1Y 4E9. patients in each group coronary sinus catheters were placed and lactate metabolism was measured as an index of ischaemia.

The striking finding of the study was the occurrence of hypotension, decreased diastolic compliance and myocardial lactate production in four patients after induction with alfentanil. One patient in the sufentanil group and none receiving fentanyl suffered these effects. Sufentanil caused a small but statistically significantly larger decrease in systolic function than the other agents. There were no differences between groups in the incidence of perioperative infarction or low cardiac output syndrome.

The authors conclude that both fentanyl and sufentanil provide haemodynamic stability during induction, although sufentanil may cause more myocardial depression and should be used cautiously in patients with impaired ventricular function. They suggest that, with the dose regimen used, alfentanil can cause haemodynamic instability and myocardial ischaemiu.

\section{End-systolic pressure-volume relation}

Contractility refers to the intrinsic ability of muscle to contract, independent of both the length to which it is initially stretched (preload) and the load it must contract against (afterload). Although an intuitively simple concept, the quantification of contractility has been difficult in isolated muscle studies as well as in vivo.

The end-systolic pressure-volume relation was plotted by Franck in $1898^{2}$ but received little attention until the early 1970's when it was shown to be a straight line. , $^{3,4}$ Grossman's showed the relation to be useful clinically in determining the inotropic state of the heart. For any constant inotropic level the ventricle will empty less completely the greater the pressure it must generate. The end-systolic pressure-volume relation is simply the line showing how much end-systolic (residual) volume increases when end-systolic pressure (afterload) increases (see Figure 1). This relationship is independent of preload and incorporates afterload into the analysis. A given 
heartbeat will arrive at end-ejection and fall on this line regardless of the starting point for end-diastolic volume and of the level of aortic pressure that it encounters during ejection. The slope of the end-systolic pressure-volume relation changes with alterations of inotropic state; if contractility is depressed the ventricle empties less completely and end-systolic volume increases at the same blood pressure (see Figure 2). This slope therefore incorporates the effect of pressure changes whereas ejection fraction, velocity of circumferential fibre shortening, and $\mathrm{dP} / \mathrm{dt}$ are substantially changed by varying afterload without any change in contractile state. Normally, to assess contractility by this method, two or three points on the line are determined using vasoactive drugs to alter blood pressure while simultaneously measuring ventricular dimensions. Since Miller et al. instead used volume loading as their intervention, the resulting small changes in the slope of the end-systolic pressure-volume relation which they report cannot be interpreted with confidence. Further evidence will be required before we can conclude that the three narcotics have different effects on myocardial contractility.

\section{Myocardial depression}

Many anaesthetic agents have been reported to cause myocardial depression. It is often unclear what is meant by this term. Miller ef al. took care to distinguish three distinct phenomena which do not necessarily occur together: changes in myocardial performance (pressurevolume work), changes in contractility, and the onset of regional myocardial ischaemia.

Myocardial performance can decrease without any change in contractility if venodilation leads to decreased venous retum and lowered cardiac output. Loss of consciousness is usually associated with reduced sympathetic tone leading to decreases in both myocardial performance and contractility. In studying a drug that induces anaesthesia, it is difficult to separate direct effects on the hear and circulation from indirect effects mediated by changes in autonomic tone.

Decreascd myocardial contractility can be infcrred from a decrease in the slope of the end-systolic pressurevolume relation. The implications of such a finding still requires an understanding of the underlying mechanism: withdrawal of sympathetic tone, decreased sarcoplasmic calcium flux, and coronary arteriolar vasodilation in a patient with coronary disease can each cause depression of contractility. The first two mechanisms may protect patients from ischaemia by reducing myocardial oxygen demand; the third mechanism would decrease contractility by aggravating myocardial ischaemia.

\section{From description to prescription}

The study by Miller et $a t$. is a detailed description of the

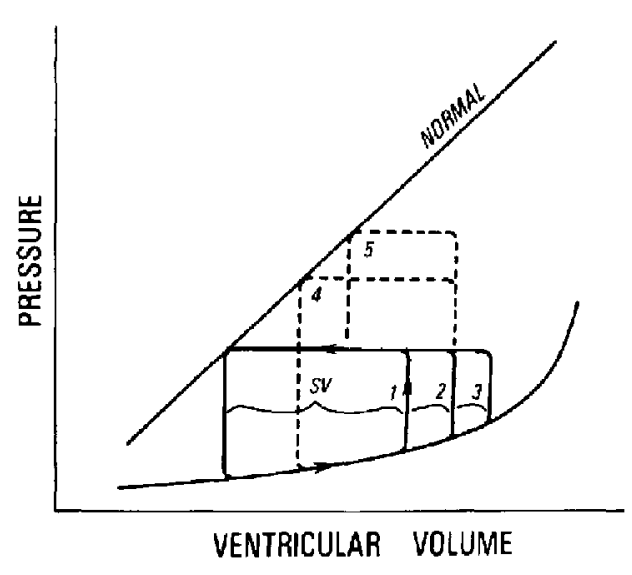

FIGURE 1 Pressure-volume loops of single contractions of the left ventricle. Reading from the bottom left comer the loop moves right as the ventricle fills during diastole, vertically upward during isovolumic contraction, left during ejection, and vertically down during isovolumic rclaxation. Beats 1, 2, and 3 show that increases in preload do not change the end-systolic point. Beats 4 and 5 show that, with contractility constant, increasing afterload causes end-systolic volume to increase and stroke yolume to decrease. (Reprinted with permission from Ross $J$ Jr. Pathophysiology of the human hear function of the heart under abnomal loading conditions. I $n$ : Krayenbuehl HP, Kuchles W, (Eds). Kardiologie in Klinik und Praxis. vol 1. Stuttgart: Georg Thieme Verlag, 1981: 31;9-31.28.)

FIGURE 1 Des courbes pression-volume lors de contractions simples du ventricule gauche. La lecure se fait à partir du bas dans le coin gauche de la courbe et la progression se fait vers la droite quand le ventricule se remplit durart la diastole, verticalement vers le haut lors de la contraction isovolémique, vers la gauche durant l'injection, et verticalement vers le bas durant la relaxation isovolémique. Les battements 1, 2 et 3 démontrent que l'augmentation dans la pré-charge ne changent pas le point de fin de systele. Les battements 4 et 5 démontrent que lors d'une contractilité constante l'augmentation de la post-charge amène une augmentation du volume en fin de systole et une diminution du volume d'éjection.

effect of three narcotics in a clinical setting. We do not know, however, if the adverse effects of alfentanil were due to interaction with other drugs, inappropriate dose or rate of administration, or if it actually differs pharmacodynamically from fentanyl and sufentanil. It is also possible that the findings were due to chance, considering the small number of patients studied with coronary sinus catheters. We must know the mechanism whereby alfentanil caused hypotension and ischaemia during the study before we can confidently predict its effects in our own practice. Description is the first step. Further studies testing causality and mechanism will be necessary before we can decide if alfentanil should be prescribed as an induction agent for patients with ischaemic heart disease. 


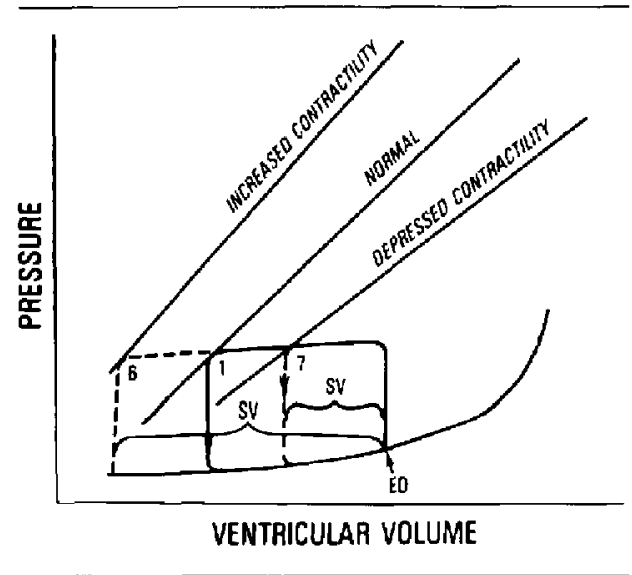

FIGURE 2 The end-systolic pressure-volume relation is shifted upward by increases in concractility. Three beats are shown originating from the same preload (arrow) and developing the same pressure during ejection. With inereased contractility (bear 6) end-systolic volume falls and stroke volume increases. With decreased contractility (beat 7 ) end-systolic volume increases and stroke volume decreases. (Reprinted with permission.)

FIGURE 2 La courbe de pression-volume en fin de systole est déplacée vers le haut lors d'une augmentation de la contractilité. Trois battements sont démontrés ayant la méme pré-charge et développant la même pression lors de l'éjection. Avec une augmentation de la contractilité (battement 6) le volume en fin te systole diminuc et le volume d'ejection augmente avec une diminution de la contractilité (batement 7) le volume en fin de systole augmente et le volume d'éjection diminue.

\section{Les narcotiques et la performance myocar- dique chez les patients atteints de maladie coronarienne}

L'étude de Miller et al.' publié dans ce numéro du Journal Canadien d'Anesthésie rapporte les résultats d'une étude clinique complexe utilisant des techniques sophistiquées chez un grand nombre de patients. Peu de chercheurs possèdent la détermination ou les ressources afin de compléter une telle étude. Les méthodes et les résultats de ce travail méritent une considération attentive par tous les anesthésistes ayant à prendre soin de patients atteints de maladie cardiaque. Je commencerai à résumer ce rappont de Miller et al. et je discuterai par la suite l'utilisation des courbes de pression-volume pour 1 'analyse de la fonction ventriculaire gauche. Le lecteur verra cette technique de plus en plus utilisée dans les travaux de recherche en anesthésie. On mettra de plus l'emphase sur la nécessité de définir soigneusement ce qu'on veut dire par "dépression myocardique." Et pour finir, on posera la question qui devra toujours être posée quand on évalue les résultats d'une étude clinique: puis-je appliquer ces résultats dans ma pratique personnelle?

L'étude a été conçue afin d'examiner les effets cardiovasculaires de l'induction anesthésique chez des patients coronariens suite à l'utilisation de trois narcotiques différents. Quatre-vingt-seize patients cédulés pour une chirurgie coronarienne élective ont reçu d'une façon randomisée soit du fentanyl, sufentanil, ou de l'alfentanil pour l'induction anesthésique. Utilisant un probe nucléaire afin d'estimer la fraction d'éjection et un cathéter dans l'artère pulmonaire afin de déterminer les débits cardiaques et en déduire le volume d'êjection, les auteurs étaient capables de calculer les volumes du ventricule gauche en fin de diastole et fin de systole. La performance myocardique était déterminée par le changement du volume d'éjection indexé suite à une augmentation du volume en fin de diastole. La fonction du ventricule gauche en systole (la capacité du ccur de se vider) est déterminée par la relation entre le volume et la pression en fin de systole (discuté plus loin).

La compliance du ventricule gauche en diastole était déterminée en traçant les changements de la pression de l'artère pulmonaire bloquée lors d'une augmentation du volume en fin de diastole. Chez neuf patients de chaque groupe des cathéters furent placés dans le sinus coronaire et le métabolisme des lactates fut mesuré comme un indice d'ischémie.

La découverte la plus frappante de cette étude fut l'hypotension, la diminution de la compliance diastolique et la production de lactate par le myocarde chez quatre patients après induction à 1 talfentanil. Les mêmes effets sont survenus chez un patient du groupe sufentanil et chez aucun des patients qui ont reçu le fentanyl. Le sufentanil a provoqué une petite diminution statistiquement significative de la fonction systolique comparativement aux autres agents. Il n'y avait aucune différence entre les groupes quant à l'incidence de l'infarcissement périopératoire ou le déclenchement du syndrome de bas débit cardiaque.

Les auteurs concluent que tant le fentanyl que la sufentanil fournissent une stabilité hémodynamique lors de l'induction même si le sufentanil peut provoquer une plus grande depression myocardique et devra ètre utilise avec précaution chez les patients avant une fonction ventriculaire altérée. Ils suggèrent qu'avec la dose uti- 
lissée, l'alfentanil peut provoquer une instabilité hémodynamique et une ischémie myocardique.

Relation pression-volume en fin de systole La contractilité se définit comme étant la capacité intrinsèque du muscle à se contracter indépendamment de la longueur à laquelle elle est étirée (pré-charge) et de la charge contre laquelle elle doit se contracter (postcharge). Même si elle est simple à concevoir, la quantification de la contractilité était difficile tant avec les études sur des muscles isolés qu'avec les études in vivo. La relation entre le volume et la pression en fin de systole fur décrite par Franck en $1898^{2}$ mais n'a attiré que peu d'attention jusqu'au début des années 1970 quand cette relation fut décrite comme variant en ligne droite. 3,4 Grossman ${ }^{5}$ a trouvé cette relation utile cliniquement afin de déterminer l'état inotrope du coeur. Pour un niveau d'inotropisme constant le ventricule se videra moins complètement si la pression qu'il doit générée est plus grande. La relation pression-volume en fin de systole est simplement la ligne qui démontre combien le volume (résiduel) en fin de systole doit algmenter quand la pression en fin de systole (post-charge) augmente (voir figure 1). Cette relation est indépendante de la pré-charge et comprend la post-charge dans son analyse. Un battement cardiaque donné arrivera en fin d'éjection et correspondra à cette ligne quelque soit le point de départ du volume en fin de diastole et du niveau de la pression aortique qu'il va rencontrer lors de l'éjection. La pente de courbe de pression-volume en fin de systole varie avec les altérations de l'inotropisme; si la contractilité est diminuée le ventricule se videra moins complètement et le volume en fin de systole augmentera pour la même pression artérielle (voir figure 2). Cette pente ainsi comprend les effets des variations de pression alors que la fraction d'éjection, la vélocité de raccourcisscment circonférentielle de la fibre, et le dP/dt sont altérés substantiellement par les changements de la post-charge sans qu'il n'v ait aucun changement de la contractilité. Normalement afin d'évaluer la contractilité par cette méthode, deux ou trois points sur cette ligne sont déterminés utilisant des substances vasoactives afin d'altérer la pression artérielle en mesurant simultanément les dimensions du ventricule. Cependant Miller et at. ont utilisé dans leur étude une charge volémique comme moyen d'obtenir ces trois points. Les changements minimes dans la pente de la relation pression-volume en fin de systole qu'ils ont rapportés ne peuvent être ainsi interprétés avee confidence. D'autres évidences sont requises avant de conclure que les trois agents narcotiques ont différents effets sur la contractilité myocardique.

\section{Dépression myocardique}

Plusieurs agents anesthésiques ont été rapportés comme responsables d'une "dcpression myocardique". Souvent il n'est pas clair ce qu'on veut dire par ce terme. Miller et al. ont pris soin de distinguer trois phénomènes distincts ne survenant pas nécessairement en même temps: les changements de la performance myocardique (travail-volumepression), les changements de la contractilité, et les débuts de l'ischémie régionale myocardique.

La performance myocardique peut diminuer sans aucun changement dans la contractilité si une vélodilatation amène une diminution de retour veineux et un bas débit cardiaque. Une perte de connaissance est habituellement associcé à une diminution du tonus sympathique provoquant une diminution de la performance myocardique et de la contractilité. En étudiant une drogue qui induit 1 'anesthésie il est diffiçile de séparer les effets directs sur le coeur et la circulation des effets indirects médiés par des changements du tonus du système autonome.

Une diminution de la contractilité myocardique peut être déduite lors d'une diminution de la pente de la courbe de la pression-volume en fin de systole. Les implications de cette trouvaille requièrent encore une compréhension du mécanisme sous-jacent: l'abolition du tonus sympathique, la diminution du flot calcique sarcoplasmique, et la vasodilatation artériolaire coronaire chez un patient atteint de maladie coronarienne peuvent être la cause de la diminution de la contractilité. Les deux premiers mécanismes peuvent protéger les patients de l'ischémie en réduisant la demande en oxygène du myocarde; le troisième mécanisme pourra diminuer la contractilité en aggravant l'ischémie myocardique.

\section{De la description à la prescription}

L'étude de Miller et al. est une description détaillée des effets de trois narcotiques dans une situation clinique. On ignore cependant si les effets adverses de l'alfentanil étaient dôs à une intéraction avec d'autres drogues, à une dose d'alfentanil ou un taux d'administration inapproprié ou une véritable différence pharmacodynamique avec le fentanyl et le sufentanil. Il est aussi possible que ces résultats soient dûs au hasard considérant le petit nombre de patients étudiés avec des carhéters dans les sinus coronaires. On doit savoir le mécanisme par lequel l'alfentanil a provoqué l'hypotension et l'ischêmie durant l'étude avant de prédire avec confiance ses effets dans notre propre pratique. La description est la première étape. D'autres études sur la causalité et le mécanisme seront nécessaires avant de décider si l'alfentanil doit être prescrit comme un agent d'induction chez les patients atteints de maladie cardiaque ischénique. 


\section{References}

1 Miller DR, Wellwood M, Teasdale SJ et al. Effects of anacsthetic induction on myocardial function and metabolism: a comparison of fentanyl, sufentanil and alfentanil. Can J Anaesth 1988; 35: 219-33.

2 Frank $O$. Die grund form des arteriellen Pulses. Z Biol 1898; 37: 483.

3 Suga $H$. Time course of left ventricular pressure-volume relationship under various extents of aortic occlusion. Jpn Heart J 1970; 11: 373.

4 Taylor RR. Covell JW, Ross J Jr. Volume-tension diagrams of ejecting and isovolumic contractions in left ventricle. Am J Physiol 1969; 216: 1097

5 Grossman W, Braunwald E, Mann $T$ et al. Contractile state of the left ventricle in man as cvaluated from endsystolic pressure-volume relations. Circulation 1977; 56: 845-52. 